\title{
FOLFIRI/Ziv-aflibercept Regimen
}

National Cancer Institute

\section{Source}

National Cancer Institute. FOLFIRI/Ziv-aflibercept Regimen. NCI Thesaurus. Code C136250.

A chemotherapy regimen consisting of leucovorin, fluorouracil and irinotecan (FOLFIRI), plus ziv-aflibercept that is used for the treatment of colon cancer. 